\title{
Rural-Urban Continuum Code 2003
}

National Cancer Institute

\section{Source}

National Cancer Institute. Rural-Urban Continuum Code 2003. NCI Thesaurus. Code C160900.

Codes published by the Office of Management and Budget (OMB) in 2003 that separate counties into four metropolitan and six non-metropolitan categories based on the size their populations and form a classification scheme that distinguishes metropolitan counties by size and non-metropolitan counties by degree of urbanization and proximity to metro areas. 